\title{
Erratum to: Long-term Effect of Ileal Transposition on Adipokine Serum Level in Zucker (Orl)-Lepr ${ }^{\text {fa }}$ Fatty Rats
}

\author{
Tomasz Sawczyn $^{1}$ • Dominika Stygar ${ }^{1}$ - Iwona Karcz-Socha ${ }^{1}$ • Jodok Fink ${ }^{3}$. \\ Bronisława Skrzep-Poloczek ${ }^{1}$ - Marcin Kłosok ${ }^{1}$ - Bogdan Doleżych ${ }^{4}$. \\ Maria Augustyniak $^{4} \cdot$ Agnieszka Zawisza-Raszka $^{4} \cdot$ Michal Kukla $^{6} \cdot$ Marek Michalski $^{5}$. \\ Aleksandra Fimiarz ${ }^{7}$ - Krystyna Żwirska-Korczala ${ }^{1}$ - Wojciech Konrad Karcz ${ }^{2}$
}

Published online: 28 August 2017

(C) Springer Science+Business Media, LLC 2017

Erratum to: Journal of Obesity Surgery (2015) 25:1848-1857

DOI 10.1007/s11695-015-1618-6

In the original article, the name of the author Wojciech Konrad

Karcz was incorrect - it is correct here.

The online version of the original article can be found at https://doi.org/ 10.1007/s11695-015-1618-6

Tomasz Sawczyn

sawczyn.t@gmail.com

1 Department of Physiology, School of Medicine with Dentistry, Division in Zabrze, Medical University of Silesia, Katowice, Poland

2 Department of Surgery, Universitäts Klinikum Schleswig Holstein, Campus Lübeck, Lübeck, Germany

3 Department of General and Visceral Surgery, Surgical Metabolic and Anastomosis Research Team SMART, Albert-Ludwigs-University, Freiburg, Germany

4 Department of Animal Physiology and Ecotoxicology, University of Silesia, Katowice, Poland

5 Department of Histology and Embryology, Faculty of Medicine and Dentistry in Zabrze, Medical University of Silesia, Katowice, Poland

6 Department of Gastroenterology and Hepatology, Medical University of Silesia, Medyków 14, 40-752 Katowice, Poland

7 Anaesthesia and Intensive Therapy Department, Maria Sklodowska-Curie Memorial Cancer Center and Institute of Oncology, Gliwice, Poland 